\title{
Quand penser à une maladie osseuse rare chez l'adulte?
}

\author{
Warning on rare bone diseases \\ Christian Roux ${ }^{\mathrm{a}, \mathrm{b}, \mathrm{c}}$ \\ aService de Rhumatologie - Hôpital Cochin, Centre National de Référence des Maladies \\ Osseuses Constitutionnelles, 27 rue du Faubourg Saint Jacques, 75014 Paris, France \\ bUniversité Paris Descartes, 75006 Paris, France \\ 'INSERM U1153, 75014 Paris, France
}

Christian Roux, Service de Rhumatologie - Hôpital Cochin, Centre National de Référence des Maladies Osseuses Constitutionnelles, 27 rue du Faubourg Saint Jacques, 75014 Paris, France christian.roux@aphp.fr 


\section{RÉSUMÉ :}

Les maladies osseuses rares ont des expressions phénotypiques variées, et certaines formes modérées peuvent être diagnostiquées à l'âge adulte. La survenue de fractures trop fréquentes ou atypiques, la constatation d'une densité osseuse effondrée ou au contraire d'une hyperdensité sont des circonstances habituelles de découverte. Mais ces maladies peuvent aussi être découvertes en raison d'enthésiopathies ossifiantes, de chondrocalcinose articulaire, de douleurs articulaires par hyperlaxité ou arthrose précoce, voire d'une polyalgie.

Le contexte familial, les antécédents personnels dans l'enfance et l'adolescence, et en particulier les problèmes dentaires vont confirmer l'hypothèse. Ces diagnostics sont utiles pour optimiser la prise en charge de ces patients, et la discussion éventuelle d'un traitement spécifique.

\section{MOTS-CLÉS :}

- Maladies osseuses rares

- Ostéogénèse imparfaite

- Hypophosphatasie

- Rachitismes

- Hyperlaxité

- Enthésiopathies. 


\section{ABSTRACT :}

Rare bone diseases have various phenotypic expression, including moderate forms diagnosed at adult age. They are associated with a high number of fractures, or atypical fractures, and bone mineral density abnormalities, either very low, or very high bone density. But clinical symptoms can be predominantly enthesiopathies, joint pain related to arthrosis and hypermobility, chondrocalcinosis, and even polyalgia. Familial and personal history can help for diagnosis, and past and current dental abnormalities are a relevant symptom in this matter.

Such diagnosis is helpful for optimal care of the patients, and new specific treatments are now available for a selected number of patients.

\section{KEY WORDS :}

- Rare bone diseases

- Osteogenesis imperfecta

- Hypophosphatasia

- Rickets

- Joint hypermobility

- Enthesiopathies. 


\section{Introduction}

L'organisation des soins en France a permis la création des centres de référence et des centres de compétence pour les maladies rares, optimisant ainsi la prise en charge des malades, en regroupant des spécialistes de toutes disciplines, organisés en réseaux. Ainsi, la Filière-SantéMaladies-Rares OSCAR, composée de 11 sites constitutifs, 71 centres de compétences, et 2 centres coordonnateurs, facilite l'orientation vers les soins appropriés des patients ayant des maladies rares de l'os, du cartilage et du métabolisme phosphocalcique. Dans la grande majorité des cas, le diagnostic est fait en pédiatrie en raison de symptômes caractéristiques. Les consultations de transition sont un moment clé, en particulier pour éviter le sentiment d'abandon, encore décrit par certains patients lorsque les soins se sont arrêtés à la fin de la croissance, ou à l'âge adulte. Les rhumatologues ont bien sûr un rôle actif dans ces consultations de transition, pour gérer les séquelles osseuses et articulaires, et les nouveaux enjeux du patient devenu adulte: autonomie fonctionnelle, douleurs, facteurs de risque associés, etc.

Mais ces maladies rares peuvent avoir une expression phénotypique très variée et certaines, létales dans leur forme la plus sévère, ont parfois des formes modérées, ainsi méconnues, et révélées à l'âge adulte. II s'agit toujours de symptômes de fragilité osseuse et/ou de douleurs de l'appareil musculo squelettique (1). Le rhumatologue est ainsi le plus souvent celui qui peut faire ce diagnostic.

Nous revoyons ici les situations cliniques qui, chez un adulte considéré jusqu'ici comme en bonne santé, peuvent faire évoquer une maladie osseuse rare.

\section{Les circonstances du diagnostic}

\subsection{Les fractures}

Le nombre, la localisation, l'évolution de certaines fractures doivent attirer l'attention.

Des fractures trop fréquentes peuvent s'observer à l'âge adulte chez les patients souffrant d'ostéogénèse imparfaite, surtout de types III ou IV, de diagnostic simple, mais peuvent aussi révéler un type I. Les fractures répétées des métatarses, consolidant mal, sont typiques de l'hypophosphatasie chez l'adulte (figure 1). Les fractures fémorales sous trochantériennes peuvent débuter par une fissure diaphysaire externe au cours de l'hypophosphatasie, au contraire des fissures des ostéomalacies « classiques » situées typiquement au bord interne du col fémoral. Ces fractures sous trochantériennes peuvent être bilatérales. Parfois s'observe seulement une 
augmentation localisée de l'épaisseur corticale diaphysaire externe. Lorsqu'elles se complètent, ces fractures ont un aspect très différent de celui des fractures fémorales ostéoporotiques : elles sont transversales, avec une faible obliquité ; le trait n'est jamais comminutif (2). De telles fractures fémorales atypiques ont été observées chez des patientes traitées par bisphosphonates en raison d'une densité osseuse basse, attribuée à une ostéoporose. Les phosphatases alcalines peuvent être basses sous traitement anti résorbeur du fait de la réduction du remodelage. Dans tous les cas c'est l'atypie des fractures qui fait vérifier a posteriori la valeur des phosphatases avant traitement ; le diagnostic est confirmé par l'analyse génétique $(3,4)$.

\subsection{Les anomalies de densité osseuse}

La constatation d'une densité osseuse très basse, en l'absence des causes habituelles d'ostéoporoses secondaires, peut être une circonstance révélatrice de maladie génétique. À l'inverse une hyperdensité osseuse peut s'observer dans les rachitismes hypophosphatémiques, la maladie de Van Buchen, les ostéopétroses et autres ostéopathies condensantes... Chez 17 patients de 35 ans en moyenne ayant un rachitisme hypophosphatémique, les $Z$ scores médians de densité rachidienne et fémorale sont $+1,9$ et $+0,9$ respectivement. Chez ces patients la densité augmente avec l'âge en parallèle de l'apparition des lésions structurales rachidiennes (5) L'hyperdensité peut toutefois exister chez des patients indemnes d'enthésiopathies, par augmentation paradoxale de quantité de tissu ostéoïde partiellement minéralisé (6).

\subsection{La découverte radiologique}

C'est une circonstance fréquente de découverte des exostoses ou d'une dysplasie fibreuse, y compris asymptomatique, sur une imagerie faite pour d'autres raisons. L'aspect parfois inquiétant d'une ostéolyse étendue ou d'une lésion osseuse hétérogène peut être source de grande inquiétude chez l'adulte jeune, et le diagnostic hasardeux de « sarcome » parfois évoqué. Les radiographies peuvent aussi révéler des séquelles de fracture, avec appositions périostées, un aspect de corticales très amincies, des déformations osseuses, des os wormiens, voire des séquelles de craniosténose (7).

\subsection{Les enthésiopathies}

L'ossification des enthèses et des ligaments vertébraux est une complication du rachitisme hypophosphatémique lié à l'X et de l'hypophosphatasie $(5,8)$. L'analyse systématique de 269 patients ayant une valeur basse de phosphatases alcalines persistante (après élimination des causes secondaires), sans hypophosphatasie génétique prouvée, et quelle que soit la raison de la consultation, montre que $43 \%$ d'entre eux ont au moins une atteinte structurale de l'appareil 
locomoteur : les enthésiopathies ossifiantes, les calcifications péri articulaires, les hyperostoses rachidiennes, sont 3,12 , et 20 fois plus fréquentes chez les femmes, 3,33 et 8 fois plus fréquentes chez les hommes par rapport à la population générale (9).

Ces ossifications expliquent en partie les valeurs très élevées de densité osseuse rachidienne mesurées en absorptiométrie biphotonique. Au cours du rachitisme hypophosphatémique, ces lésions sont quasi identiques à celles observées au cours de la spondylarthrite (figure 2). Ces patients ont des douleurs articulaires et rachidiennes parfois de rythme inflammatoire, des anomalies (parfois une fusion) des sacro iliaques, des enthésiopathies ossifiantes du bassin, des syndesmophytes rachidiens... et plusieurs cas de patients ainsi traités par anti TNF pour « spondylarthrite » (sans succès) ont été rapportés $(10,11)$. La fréquence des enthésiopathies augmente avec l'âge $(80 \%$ des patients de plus de 40 ans $(5,12))$. L'analyse systématique de l'ensemble du squelette de 52 patients âgés de 42 ans en moyenne ayant un rachitisme hypophosphatémique, montre la présence d'enthésiopathies chez $64 \%$ d'entre eux ; des fissures osseuses sont visibles chez $36 \%$ d'entre eux (13). Les enthésiopathies sont la cause la plus importante de douleurs et d'altération de qualité de vie chez les patients adultes ayant un rachitisme hypophosphatémique (13). Ces observations démontrent que des lésions structurales considérées comme caractéristiques de spondylarthrite peuvent survenir sans lésion inflammatoire sous-jacente, et sont dues aux contraintes mécaniques sur matrice osseuse anormale.

\subsection{La chondrocalcinose articulaire}

La chondrocalcinose est une complication de l'hypophosphatasie. La phosphatase alcaline hydrolyse le pyrophosphate extra cellulaire (issu des nucléotides triphosphates et du transport du pyrophosphate inorganique intra cellulaire par ANK (progressive ankylosis protein homolog)), en présence de magnésium, pour former l'orthophosphate. La diminution de quantité ou d'activité enzymatique cause un excès de pyrophosphate. Le risque d'avoir une chondrocalcinose est 7 à 8 fois plus important chez les patients ayant des phosphatases alcalines basses persistantes, et est constaté chez 20 à $40 \%$ de ces patients $(8,9,14)$. Chez certains patients sont observés aussi des dépôts périarticulaires d'apatite. Une anomalie du gène de la phosphatase alcaline a été décrite chez 3 sœurs ayant des calcifications tendineuses multiples, sans chondrocalcinose et sans fragilité osseuse (15).

\subsection{Le syndrome polyalgique}

Le syndrome polyalgique peut être au premier plan, et la raison de la consultation. Paresthésies, crampes, malaises, font évoquer l'hypocalcémie de l'hypoparathyroïdie. La fatigue musculaire est rapportée chez $40 \%$ des patients ayant une ostéogénèse imparfaite $(1,7)$. Des troubles de la nociception sont très probables au cours de l'hypophosphatasie, au cours de 
laquelle l'anxiété et la dépression sont plus fréquentes que dans la population générale, justifiant les recherches en cours sur le rôle de la phosphatase alcaline dans le fonctionnement du système nerveux central (8). Les anomalies de composition corporelle, avec rapport masse grasse/masse maigre anormal sont étudiées dans le rachitisme hypophosphatémique lié à l'X (12).

Les patients préoccupés par la fragilité osseuse et les douleurs articulaires ne décrivent pas toujours spontanément le syndrome douloureux : douleurs musculaires, céphalées sont pourtant fréquentes lors d'interrogatoires systématiques dans la plupart des maladies osseuses constitutionnelles. Les causes de ces douleurs ne sont sans doute pas les mêmes dans ces différentes maladies.

\subsection{L'hyperlaxité}

Elle est fréquente dans beaucoup de maladies génétiques (syndrome de l'X fragile, maladie de Morquio, trisomie 21...). C'est une complication de l'ostéogénèse imparfaite (7) : le recurvatum des genoux expose à ses propres complications : instabilité articulaire, chutes, fissures osseuses secondaires aux contraintes mécaniques locales anormales. Les ruptures tendineuses peuvent concerner jusqu'à $40 \%$ des patients d'une association de malades souffrant d'ostéogénèse imparfaite tous types confondus et répondant à un questionnaire (1). Le syndrome d'Ehlers-Danlos non vasculaire (ancien type III dit hypermobile) associe l'hypermobilité articulaire (responsable d'entorses et subluxations fréquentes) et des signes cutanés : hyperextensibilité (faces antérieures des avant-bras et faces latérales du cou), retards de cicatrisation, ecchymoses fréquentes, cicatrices dystrophiques. Ces patients peuvent avoir des densités osseuses basses. Ce syndrome rare se distingue du syndrome d'hypermobilité articulaire bénigne, fréquent, par l'association à d'autres symptômes (polyalgie, asthénie, crampes, signes de dysautonomie). II est toutefois indispensable de faire le diagnostic sur les signes articulaires et cutanés, et pas seulement sur une accumulation de manifestations fonctionnelles, en raison du risque de confusion avec une fibromyalgie, ou une association fortuite de symptômes très fréquents (16).

La recherche systématique d'une hypermobilité articulaire et le calcul du score de Beighton (tableau 1) sont utiles dans ces circonstances. Ce score est calculé à partir de manœuvres cliniques, comptant chacune pour 1 point. Chez l'adulte un score supérieur ou égal à 5 (sur un maximum de 9) fait retenir le diagnostic d'hyperlaxité. Le score de Bulbena teste 9 articulations et un point supplémentaire est accordé en cas d'ecchymoses (17). L'hyperlaxité diminue avec l'âge, y compris parfois chez les patients souffrant d'Ehlers-Danlos, du fait d'arthroses précoces. L'interrogatoire recherche alors les signes manifestes à l'enfance et l'adolescence. 


\section{Les signes à rechercher}

Lorsque les circonstances cliniques ont permis d'évoquer l'hypothèse d'une maladie osseuse ou métabolique rare, certains symptômes doivent être recherchés.

\subsection{Les anomalies squelettiques}

L'interrogatoire peut retrouver des fractures ou fissures fréquentes dans l'enfance et l'adolescence (14). Les incurvations des os longs, les déformations du sternum (thorax en carène, pectus carinatum, saillie du sternum...), la scoliose, sont fréquentes au cours de l'ostéogénèse imparfaite, de l'hypophospatasie. Au cours du rachitisme hypophosphatémique lié à l'X, la déformation séquellaire des membres inférieurs chez l'adolescent est en valgus, et souvent asymétrique.

\subsection{Les anomalies dentaires}

Fréquemment oubliées à l'interrogatoire et l'examen clinique, elles sont pourtant un signe majeur d'alerte et d'orientation dans ce contexte de maladies des tissus minéralisés.

La chute prématurée des dents déciduales (avec les racines), les anomalies de l'émail, les pertes de dents spontanées par défaut de cément, ou par avulsions fréquentes, les difficultés après pose d'implants, les caries répétées, etc... sont courantes chez ces patients et doivent être systématiquement recherchées, car les patients n'en parlent quasi-jamais spontanément. Les complications infectieuses sont fréquemment observées chez les patients ayant des rachitismes hypophosphatémiques (18). Les anomalies de structure et de couleur (par atteinte de la dentine ou de l'émail) peuvent passer inaperçues.

\subsection{Les autres anomalies}

La néphrocalcinose, et les lithiases rénales sont plus fréquentes qu'attendues au cours de l'hypophosphatasie et de l'hypoparathyroïdie. Les sclérotiques sont de couleur bleue plus ou moins foncée chez $80 \%$ des patients ayant une ostéogénèse imparfaite, et les déficits auditifs (otospongiose) souvent associés (environ $40 \%$ des patients adultes). Certaines rétinopathies s'accompagnent de fragilité osseuse (syndrome de Stickler). 


\subsection{Les examens biologiques}

Ils sont par définition sans particularité au cours des maladies osseuses constitutionnelles.

Le retard diagnostique de la plupart des maladies du métabolisme phosphocalcique, souvent de plusieurs années ou dizaines d'années après l'apparition du premier signe peut être dû à l'absence de prescription d'examen biologique, mais bien plus souvent à l'absence d'analyse du résultat. Les hypocalcémies sont négligées si elles ne s'accompagnent pas de malaises ou tétanie. Les hypophosphatémies ne sont pas souvent considérées comme responsables des symptômes musculo squelettiques. L'anomalie recherchée des phosphatases alcalines est leur augmentation, signe de maladie de Paget ou d'ostéomalacie, mais l'hypophosphatasémie passe totalement inaperçue. La prévalence de l'hypophosphatasie en milieu hospitalier est de 0,13\%; l'analyse des données des patients ayant une telle anomalie biologique (persistance sur plusieurs examens) montre qu'elle n'est quasiment jamais signalée dans le compte rendu d'hospitalisation (19).

\section{Conclusion}

La reconnaissance de ces maladies rares est importante pour éviter des errements, des prises en charge incorrectes, des médicaments inadaptés. Une valeur basse des phosphatases alcalines en particulier doit être recherchée avant de débuter un traitement anti résorbeur. Le diagnostic permet d'inscrire le patient dans un parcours de soins adapté, incluant le conseil génétique, et d'optimiser les soins prodigués par les autres spécialistes (dentistes, chirurgiens...). L'arrivée des traitements dans l'hypophosphatasie (asfotase alfa), et le rachitisme hypophophatémique lié à l'X (burosumab), et les essais en cours dans d'autres maladies rares (fibrodysplasie ossifiante), ouvrent l'ère des thérapeutiques des maladies osseuses rares, d'efficacité spectaculaire pour ces patients.

Déclaration de liens d'intérêts

Honoraires pour interventions ponctuelles : Alexion, Amgen, UCB

Subvention pour Association de Recherche ; Ultragenyx. 


\section{RÉFÉRENCES:}

1. Mc Kiernan FE. Musculoskeletal manifestations of mild osteogenesis imperfecta in the adult. Osteoporos Int 2005; 16:1698-1702.

2. Maman E, Briot K, Roux C. Atypical femoral facture in a 51-year woman revealing a hypophosphatasia. Joint Bone Spine 2016; 83:346-348.

3. Sutton RA, Mumm S, Coburn SP, Ericson KL, Whyte MP. Atypical femoral fractures during bisphosphonate exposure in hypophosphatasia. J Bone Miner Res 2012; 27:987-994.

4. Whyte MP. Atypical femoral fractures, bisphosphonates and adult hypophosphatasia. J Bone Miner res 2009; 24:1132-1134.

5. Beck-Nielsen SS, Brusgaard K, Rasmussen LM, Brixen K, Brock-Jacobsen BB, Poulsen MR et al. Phenotype presentation of hypophosphatemic rickets in adults. Calcif Tissue 2010; 87:108119.

6. Marie PJ, Glorieux FH. Bone histomorphetry in asymptomatic adults with hereditary hyphophosphatemic vitamin D-resistant osteomalacia. Metab Bone Dis Res 1982; 4:249-253.

7. Forlino A, Marini JC. Osteogenesis imperfecta. Lancet 2016; 387:1657-1671.

8. Briot K, Roux C. Adult hypophosphatasia. Curr Opin Rheumatol 2016; 448-451.

9. McKiernan FE, Berg RL, Fuehrer J. Clinical and radiographic findings in adults with persistent hypophosphatasemia. J Bone Miner Res 2014; 29:1651-1660.

10. Garip Y, Dedeoglu M, Bodur H. Osteomalacia mimicking spondyloarthropathy: a case report. Osteoporos Int 2014; 25:1983-1985.

11. Silvas F, Yurdakul FG, Durak M, Hatipglu G, Omal ED, Bodur H. Hypophosphatemic osteomalacia: a case simulating ankylosing spondylitis treated with anti-TNF therapy. Osteoporos Int 2016; 27:3651-3654.

12. Linglart A, Biosse-Duplau M, Briot K, Chaussain C, Esteile C, Guillaume-Czitrom S et al. Therapeutic management of hypophosphatemic rickets from infancy to adulthood. Endocr Connect $2014 ; 3: 13-30$. 
13 Che H, Roux C, Etcheto A, Rothenbuhler A, Kamenicky P, Linglart A, Briot K. Impaired quality of life in adults with $X$-linked hypophosphatemia and skeletal symptoms. European Journal of Endocrinology 2016; 174: 325-333.

14 Berkseth, Tebben PJ, Drake MT, Hefferan TE, Jewison, Wermers R. Clinical spectrum of hypophosphatasia in adults. Bone 2013; 54:21-27.

15 Guanabens N, Mumm S, Möller I, Gonzalez-Roca E, Peris P, Demertzis L, Whyte MP. Calcific periarthritis as the only clinical manifestation hypophosophatasia in middle-aged sisters. $\mathrm{J}$ Bone Miner Res 2014; 29:929-934.

16 Castori M, Camerota F, Celletin C et al. Natural history and manifestation of the hypermobility type Elhers-Danlos syndrome: a pilot study on 21 patients. Am J Med Genet 2010 ; $152 \mathrm{~A}: 556-564$.

17 Bulbena A, Duro JC, Porta M, Faus S, Vallescar R, Martin-Santos R. Clinical assessment of hypermobility of joints: assembling criteria. J. Rheumatol 1992; 19: 115-122.

18 Chaussain-Miller C, Sinding C, Wolikow M, Lasfargues JJ, Godeau G, Garabedian M. Dental abnormalities in patients with familial hypophosphatemic vitamin-D resistant rickets: prevention by early treatment with 1-hyroxyvitamine D. J Pediatr 2003; 142:324-331.

19 Maman E, Borderie D, Roux C, Briot K. Absence of recognition of low alkaline phosphatse level in a tertiary care hospital. Osteoporos Int 2016; 27:1251-1254. 
FIGURE 1: Hypophosphatasie diagnostiquée à 51 ans: fracture diaphysaire fémorale, et fractures avec pseudarthrose des métatarses (référence 2)

FIGURE 2 : Rachitisme hypophosphatémique lié à I'X : aspect de syndesmophyte en L2-L3, fusion des sacroiliaques, enthésiopathies du bassin 
TABLEAU 1: score de l'hypermobilité articulaire de Beighton

\begin{tabular}{lcc}
\hline \multicolumn{1}{c}{ Manœuvre } & Négative & Positive \\
\hline Dorsiflexion passive du $5^{\text {ème }}$ doigt $>90^{\circ}$ & 0 & 1 (unilatéral), 2 (bilatérale) \\
\hline Flexion passive du pouce vers l'avant-bras & 0 & 1 (unilatéral), 2 (bilatérale) \\
\hline Hyperextension $>10^{\circ}$ du coude & 0 & 1 (unilatéral), 2 (bilatérale) \\
\hline Hyperextension $>10^{\circ}$ des genoux & 0 & 1 (unilatéral), 2 (bilatérale) \\
\hline $\begin{array}{l}\text { Flexion complète du tronc, genoux complétement } \\
\text { étendus, avec les paumes complètement sur le sol }\end{array}$ & 0 & 1 \\
\hline
\end{tabular}

Le diagnostic de syndrome d'Elhers-Danlos peut être évoqué lorsque le score est supérieur ou égal à 5 . 



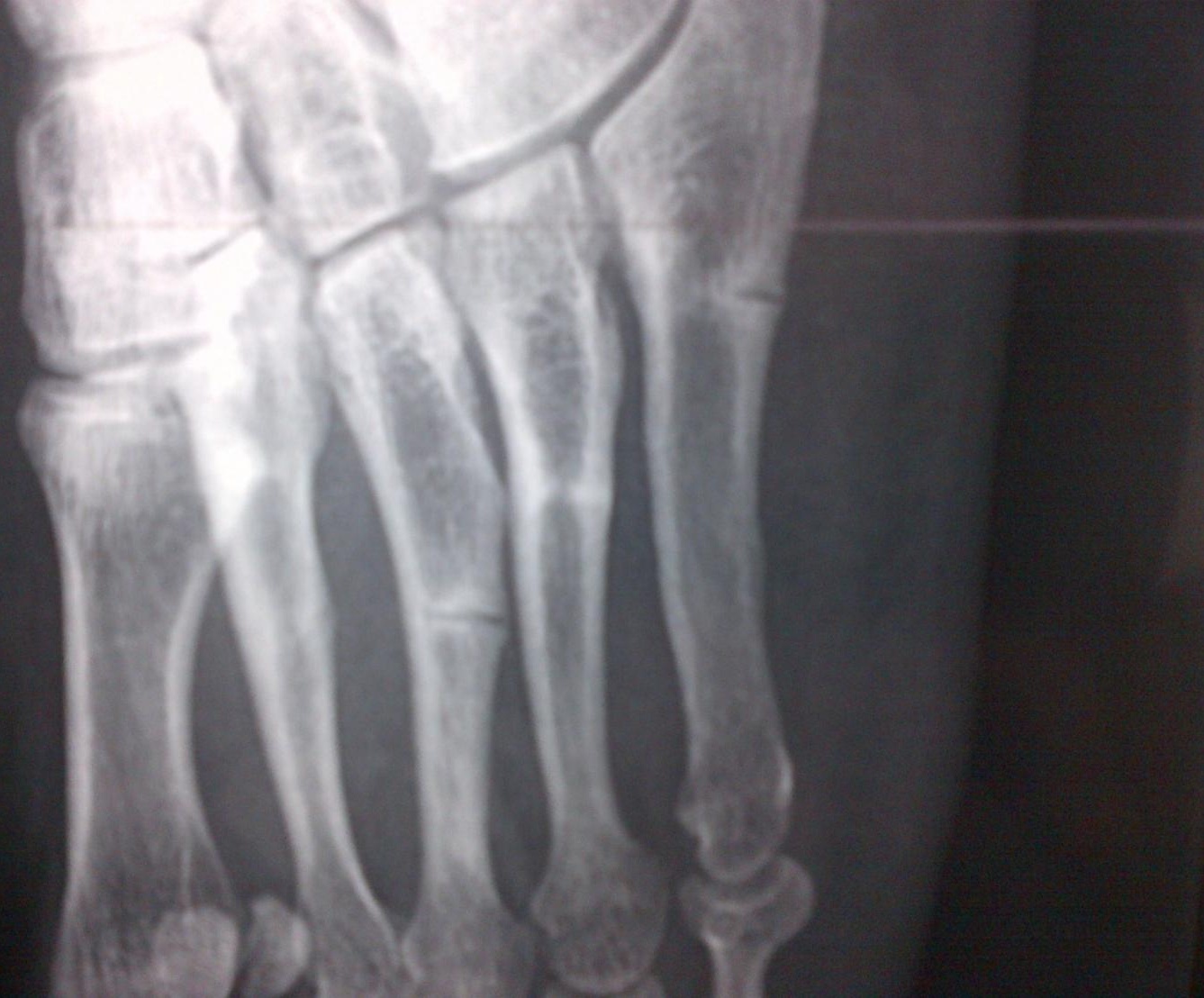



D

G

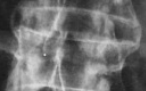
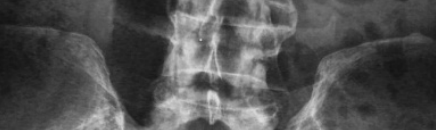

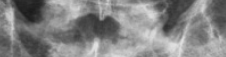
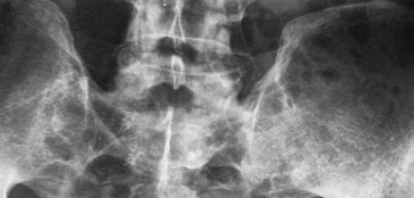

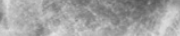

